\title{
More reverse mathematics of the Heine-Borel Theorem
}

\author{
JEFFRY L HIRST \\ JESSICA MILLER
}

\begin{abstract}
Using the techniques of reverse mathematics, we characterize subsets $X \subseteq[0,1]$ in terms of the strength of $\operatorname{HB}(X)$, the Heine-Borel Theorem for the subset. We introduce $W(X)$, formalizing the notion that the Heine-Borel Theorem for $X$ is weak, and $\mathrm{S}(X)$, formalizing the notion that the theorem is strong. Using these, we can prove the following three results: $\mathrm{RCA}_{0} \vdash \mathrm{W}(X) \rightarrow \mathrm{HB}(X)$, $\mathrm{RCA}_{0} \vdash \mathrm{S}(X) \rightarrow\left(\mathrm{HB}(X) \rightarrow \mathrm{WKL}_{0}\right)$, and $\mathrm{ATR}_{0} \vdash \bar{X}$ exists $\rightarrow(\mathrm{W}(X) \vee \mathrm{S}(X))$.
\end{abstract}

2010 Mathematics Subject Classification 03B30, 03 F35 (primary); 03F60, 26E40 (secondary)

Keywords: reverse mathematics, Heine-Borel, compact, countable closed, derived sequence, rca, wkl, aca, atr

One of the earliest results involving $\mathrm{WKL}_{0}$ is Friedman's theorem showing the equivalence of $\mathrm{WKL}_{0}$ and the Heine-Borel Theorem for [0,1]. (See Friedman's abstracts [2].) In response to a question of Friedman, Hirst [4] shows that the Heine-Borel Theorem for closed subsets of $\mathbb{Q} \cap[0,1]$ is also equivalent to $\mathrm{WKL}_{0}$. This paper extends this work, giving a characterization of those subsets of $[0,1]$ for which the Heine-Borel Theorem is equivalent to $\mathrm{WKL}_{0}$ and those for which it is not.

Our results make use of four of the "big five" axiom systems of reverse mathematics: $R C A_{0}, W K L_{0}, A C A_{0}$, and $A T R_{0}$. Extensive material related to the axiomatization and application of these subsystems to the study of complete separable metric spaces can be found in Simpson's book [5]. (See especially sections II.4 and II.5.) For example, open subsets of $[0,1]$ can be encoded as unions of sequences of open intervals with rational endpoints. Closed subsets can be encoded as the complements of open sets. The codes for open sets and closed sets are sets of natural numbers, and thus elements of models of second order arithmetic. We can also consider subsets of $[0,1]$ defined by formulas of second order arithmetic. Such sets may have countable encodings (e.g. as closed sets or analytic sets do) or they may lack specified encodings. Due to the structure of our results, they hold for definable subsets of $[0,1]$. For example, consider the following scheme formalizing the Heine-Borel Theorem for subsets of $[0,1]$. 
$\mathrm{HB}(X): X$ is a subset of $[0,1]$ and for every closed (in the topology on $\mathbb{R}$ ) subset $A \subseteq X$, every open cover of $A$ contains a finite subcover.

In the preceding statement, $X$ is an arbitrary definable subset of $[0,1]$ with no specified encoding, while the closed set $A$ has the usual countable code. A full formalization of $\mathrm{HB}(X)$ would depend on the defining formula of $X$ as in the following: If $A$ is a closed subset of the reals and every real $\alpha \in A$ satisfies the defining formula of $X$, then every open cover of $A$ contains a finite subcover.

We will present two properties of subsets, $\mathrm{W}(X)$ and $\mathrm{S}(X)$, such that $\mathrm{RCA}_{0} \vdash \mathrm{W}(X) \rightarrow$ $\mathrm{HB}(X), \mathrm{RCA}_{0} \vdash \mathrm{S}(X) \rightarrow\left(\mathrm{HB}(X) \rightarrow \mathrm{WKL}_{0}\right)$, and $\mathrm{ATR}_{0} \vdash \bar{X}$ exists $\rightarrow(\mathrm{W}(X) \vee \mathrm{S}(X))$. We can think of $\mathrm{W}(X)$ as affirming that the Heine-Borel Theorem for $X$ is weak; $\mathrm{HB}(X)$ can be proved in $\mathrm{RCA}_{0} . \mathrm{S}(X)$ indicates that the Heine-Borel Theorem for $X$ is stronger; $W_{K} L_{0}$ can be deduced from $\mathrm{HB}(X)$.

One might be tempted to use the formulas $\mathrm{HB}(X) \rightarrow \mathrm{WKL}_{0}$ for $\mathrm{S}(X)$ and $\neg(\mathrm{HB}(X) \rightarrow$ $\mathrm{WKL}_{0}$ ) for $\mathrm{W}(X)$, but these formulations are poor characterizations. For example, when $X$ is a finite collection of standard rational numbers, $\mathrm{HB}(X)$ is true in every model of $\mathrm{RCA}_{0}$, but $\neg\left(\mathrm{HB}(X) \rightarrow \mathrm{WKL}_{0}\right)$ fails in all of those models of $\mathrm{RCA}_{0}$ in which $\mathrm{WKL}_{0}$ holds. Our choices for $\mathrm{W}(X)$ and $\mathrm{S}(X)$ address this issue, as shown by Theorem 5 .

Empirically, the deduction of $\mathrm{WKL}_{0}$ from a version of the Heine-Borel Theorem is generally carried out via some construction paralleling that of the Cantor middle third set. Consequently, it seems reasonable that $\mathrm{S}(X)$ should assert that the closure of $X$ contains a perfect set. A perfect set is closed and dense in itself. A set is dense in itself if every element is an accumulation point, that is, if every neighborhood of a point $x$ contains a point of the set that is distinct from $x$. Since Brown [1] showed that $\mathrm{ACA}_{0}$ is required to prove the existence of separable closures of sets, we will use the following formulation to sidestep reference to closures.

$\mathrm{S}(X): X$ is a subset of $[0,1]$ and there is a countable dense in itself set $Y$ which is contained in every closed superset of $X$.

In strong enough subsystems, if the closure of $X$ contains no perfect set, then it is countable and its derived sequence can be used to construct a homeomorphism between the closure of $X$ and a well-ordered space. In $\mathrm{RCA}_{0}$ we can capture that situation as follows.

$\mathrm{W}(X): X$ is contained in a countable closed subset of $F \subseteq[0,1]$ and there are functions $f$ and $g$ and a well ordering $Y$ satisfying:

- The function $f: F \rightarrow Y$ is one to one. 
- For any $b_{1}, b_{2} \in(\mathbb{Q} \cap[0,1]) \cup\{-.1,1.1\}$ with $b_{1}<b_{2}$, if $F-\left(b_{1}, b_{2}\right)$ is nonempty, then the value of $g\left(b_{1}, b_{2}\right)$ is an element of $F-\left(b_{1}, b_{2}\right)$ such that

$$
\forall x \in F-\left(b_{1}, b_{2}\right) f(x) \leq f\left(g\left(b_{1}, b_{2}\right)\right) .
$$

Naïvely, $\mathrm{W}(X)$ says that $F$ can be well ordered in such a way that many closed subsets of $F$ (and hence of $X$ ) have a maximum element. For closed sets that are complements of open intervals with rational endpoints, the function $g$ selects that maximum. Appending -.1 and 1.1 to the list of possible endpoints allows us to consider open intervals containing 0 and 1 .

With our conditions formalized, we can prove the first two theorems verifying that our characterization of the strength of $\mathrm{HB}(X)$ is successful.

Theorem $1\left(\mathrm{RCA}_{0}\right)$ If $\mathrm{W}(X)$ holds, then the Heine-Borel Theorem holds for $X$. That is, $\mathrm{W}(X)$ implies $\mathrm{HB}(X)$.

Proof Suppose $\mathrm{W}(X)$ holds and fix a closed $A \subseteq X$. Let $F, Y, f$, and $g$ be provided as in the statement of $\mathrm{W}(X)$. (Note that this proof makes no further mention of $X$. All the remaining sets are encoded by subsets of $\mathbb{N}$.) Let $\mathcal{U}=\left\langle U_{n}\right\rangle_{n \in \mathbb{N}}$ be an open cover of $A$. Since $A$ is closed, we may append the complement of $A$ to $\mathcal{U}$, extending $\mathcal{U}$ to a cover of $F$. Since each open set is encoded as a union of open intervals with rational endpoints (see section II.4 of Simpson's book [5]), without loss of generality we may assume that each $U_{n}$ is an open interval with endpoints in $(\mathbb{Q} \cap[0,1]) \cup\{-.1,1.1\}$. We will define a sequence of open intervals and a sequence of elements of $A$ simultaneously. Let $V_{0}$ be the empty interval $(1.1,1.1)$. Suppose that $V_{n}$ has been defined. If $\cup_{j \leq n} V_{j} \supseteq A$, then stop. Otherwise, let $a_{n}=\min \left\{g\left(V_{j}\right) \mid j \leq n\right\}$. Let $V_{n+1}$ be the first occurring element of $\mathcal{U}$ that contains $a_{n}$. This process must halt, since otherwise $\left\langle f\left(a_{n}\right)\right\rangle_{n \in \mathbb{N}}$ is an infinite descending sequence in $Y$. Thus for some $n, \cup_{j \leq n} V_{j} \supseteq A$, so $A$ is covered by $n$ elements of $\mathcal{U}$.

Theorem 2 (RCA $)$ If $\mathrm{S}(X)$ holds, then the Heine Borel theorem for $X$ implies $\mathrm{WKL}_{0}$. That is, $\mathrm{S}(X)$ implies $\mathrm{HB}(X) \rightarrow \mathrm{WKL}_{0}$.

Proof Suppose $S(X)$ holds and the Heine-Borel theorem holds for every closed subset of $X$. Let $Y$ be as described in $\mathrm{S}(X)$. (Note that this proof makes no further mention of $X$. All the remaining sets are encoded by subsets of $\mathbb{N}$.) First we will construct several sequences indexed by elements of $2^{<\mathbb{N}}$. Roughly, the following is a generalized form of the Cantor middle third construction. Each $M_{\sigma}$ is analogous to a middle third interval 
and each $A_{\sigma}$ corresponds to a left or right interval. Let $A_{\langle\rangle}$be the open interval $(0,1)$, $P_{\langle\rangle}=(-0.1,1.1)$, and pick $y_{\langle\rangle} \in Y \cap A_{\langle\rangle}$. Suppose that we have constructed $A_{\sigma}, P_{\sigma}$, and $y_{\sigma}$ such that $A_{\sigma}$ and $P_{\sigma}$ are both open intervals with rational endpoints, $\overline{A_{\sigma}} \subseteq P_{\sigma}$, $y_{\sigma} \in A_{\sigma} \cap Y$, and $\left|A_{\sigma}\right| \leq 2^{-\operatorname{lh}(\sigma)}$. (Note that since $A_{\sigma}$ is an open interval with rational endpoints, $\mathrm{RCA}_{0}$ proves that $\bar{A}_{\sigma}$ exists. We use similar closures throughout the proof as notational conveniences.) Since $Y$ is dense in itself, we may choose distinct points $y_{\sigma \frown 0}$ and $y_{\sigma^{\wedge} 1}$ in $A_{\sigma} \cap\left(Y-\left\{y_{\sigma}\right\}\right)$. Let $a$ and $b$ denote the endpoints of $A_{\sigma}$. Let $d$ be the minimum of $2^{-\mathrm{lh}(\sigma)}$ and the distances between every distinct pair of points in $\left\{a, b, y_{\sigma}, y_{\sigma^{\wedge} 0}, y_{\sigma^{\wedge} 1}\right\}$. For $i \in\{0,1\}$, let $A_{\sigma^{\wedge} i}$ be an open interval centered at $y_{\sigma^{\wedge} i}$ with a radius of at most $d / 3$ and let $P_{\sigma^{\wedge} i}$ be an open interval around $y_{\sigma \frown i}$ with a radius of $0.4 d$. Set $M_{\sigma}=P_{\sigma}-\left(\bar{A}_{\sigma \frown 0} \cup \bar{A}_{\sigma \wedge 1}\right)$, and note that $M_{\sigma}$ is a union of three open intervals with rational endpoints. Finally, since $Y$ is a subset of every closed superset of $X$, we may choose $x_{\sigma} \in X$ such that $\left|x_{\sigma}-y_{\sigma}\right|<d / 3$.

Intuitively, the reals $\left\{x_{\sigma} \mid \sigma \in 2^{\mathbb{N}}\right\}$ behave like the midpoints of the middle third intervals used to build the Cantor set. Consider the following claim.

Claim Let $x \in[0,1]$. The real $x$ is an accumulation point of $\left\{x_{\sigma} \mid \sigma \in 2^{\mathbb{N}}\right\}$ if and only if for every $\sigma$, if $x \in \bar{A}_{\sigma}$ then $x \in \bar{A}_{\sigma \frown 0}$ or $x \in \bar{A}_{\sigma \wedge 1}$.

Proof of the claim: To prove the reverse implication, suppose that for every $\sigma$, if $x \in \bar{A}_{\sigma}$ then $x \in \bar{A}_{\sigma^{\wedge} 0}$ or $x \in \bar{A}_{\sigma^{\wedge} 1}$. Thus we have a sequence of rapidly shrinking neighborhoods $A_{\sigma_{i}}$, each of which contain $x$. The associated sequence of distinct reals $\left\langle x_{\sigma_{i}}\right\rangle_{i \in \mathbb{N}}$ converges to $x$.

To prove the forward implication, we will prove the contrapositive. Suppose there is a $\sigma$ such that $x \in \bar{A}_{\sigma}$ but $x \notin\left(\bar{A}_{\sigma \sim 0} \cup \bar{A}_{\sigma \sim 1}\right)$. Either $x=x_{\sigma}$ or $x \neq x_{\sigma}$. Then either $A_{\sigma}-\left(\bar{A}_{\sigma^{\frown} 0} \cup \bar{A}_{\sigma^{\frown} 1}\right)$ or $P_{\sigma}-\left(\bar{A}_{\sigma^{\frown} 0} \cup \bar{A}_{\sigma^{\frown} 1}\right)$, respectively, is an open set containing $x$ and no other element of $\left\{x_{\sigma} \mid \sigma \in 2^{\mathbb{N}}\right\}$. So $x$ is not an accumulation point of $\left\{x_{\sigma} \mid \sigma \in 2^{\mathbb{N}}\right\}$, completing the proof of the claim.

Finally, we turn to the actual deduction of $\mathrm{WKL}_{0}$ from the Heine-Borel Theorem. Let $T$ be a $0-1$ tree with no infinite paths. We will show that $T$ is finite. Let $C=\left\{x_{\sigma} \mid \sigma \in T\right\}$ and let $U$ be the open set consisting of the union of $\left\{M_{\sigma}-\left\{x_{\sigma}\right\} \mid \sigma \in 2^{\mathbb{N}}\right\}$ and $\left\{P_{\sigma^{\frown} i} \mid \sigma \in T \wedge i \in\{0,1\} \wedge \sigma^{\wedge} i \notin T\right\}$. We want to show that $\mathrm{C}$ is closed by showing that $\mathrm{C}$ is the complement of $\mathrm{U}$. For each $x \in[0,1]$, we need to show $x \in C$ or $x \in U$ but not both. We consider two cases.

Case I: The real $x$ is not an accumulation point of $\left\{x_{\sigma} \mid \sigma \in 2^{\mathbb{N}}\right\}$. In this case, by the claim there is some $\sigma$ such that $x \in \bar{A}_{\sigma}$ but $x \notin\left(\bar{A}_{\sigma \frown 0} \cup \bar{A}_{\sigma \frown 1}\right)$. Note that $x \in P_{\sigma}$ since 
$x \in \bar{A}_{\sigma}$. So $x \in M_{\sigma}=P_{\sigma}-\left(\bar{A}_{\sigma \frown 0} \cup \bar{A}_{\sigma \sim 1}\right)$. So $x=x_{\sigma} \in C$ or $x \in M_{\sigma}-\left\{x_{\sigma}\right\} \subseteq U$. Note that $M_{\sigma} \cap C=\left\{x_{\sigma}\right\}$, so $x$ is in exactly one of $C$ and $U$.

Case II: The real $x$ is an accumulation point of $\left\{x_{\sigma} \mid \sigma \in 2^{\mathbb{N}}\right\}$. In this case, note that $x \notin C$, since when $x \in C$, the open set $A_{\sigma}-\left(\bar{A}_{\sigma \frown 0} \cup \bar{A}_{\sigma \wedge 1}\right)$ contains no other element of $\left\{x_{\sigma} \mid \sigma \in 2^{\mathbb{N}}\right\}$. We need to show that $x \in U$. So by the claim, if $x \in \bar{A}_{\sigma}$ then $x \in \bar{A}_{\sigma^{\wedge} 0}$ or $x \in \bar{A}_{\sigma^{\wedge} 1}$. Note that $x \in \bar{A}_{\langle\rangle}$. So we can build a sequence of $\left\langle\bar{A}_{\sigma_{i}}\right\rangle_{i \in \mathbb{N}}$ where $x$ is in each $\bar{A}_{\sigma_{i}}$. This sequence of $\left\langle\sigma_{i}\right\rangle_{i \in \mathbb{N}}$ represents a path. Our tree $\mathrm{T}$ has no infinite paths, so there is some $\sigma_{j}$ such that $\sigma_{j} \in T$ but $\sigma_{j} i \notin T$ for $i \in\{0,1\}$. Note that for some $i, \bar{A}_{\sigma_{j} \wedge i}$ is in our sequence, so $x \in \bar{A}_{\sigma_{j} \wedge i}$. Thus $x \in P_{\sigma_{j} \wedge i}$. Consequently, $x \in\left\{P_{\sigma^{\wedge} i} \mid \sigma \in T \wedge i \in\{0,1\} \wedge \sigma^{\wedge} i \notin T\right\} \subseteq U$.

So in each of our cases, $x \in C$ or $x \in U$ but not both. We may conclude that $C$ is closed. Consider the open cover of $C$ given by $V=\left\{M_{\sigma} \mid \sigma \in T\right\}$. Since the Heine-Borel Theorem holds for $\mathrm{C}$, then $\mathrm{C}$ is covered by a finite subset $V_{0}$ of $V$. Each element of $V_{0}$ contains exactly one element of $C$, so $C$ is finite. Each sequence in $T$ corresponds to exactly one element of $C$, so $T$ is finite. This completes the proof of the contrapositive of $\mathrm{WKL}_{0}$.

Our next goal is to prove that exactly one of $\mathrm{W}(X)$ and $\mathrm{S}(X)$ holds for each reasonable choice of $X$. This proof will require $\mathrm{ATR}_{0}$, and we prepare with some technical discussions of perfect sets and derived sequences.

Lemma $3\left(\mathrm{ACA}_{0}\right)$. Every perfect subset of $[0,1]$ contains a countable dense in itself set.

Proof Suppose $C$ is a perfect subset of $[0,1]$. Since $C$ is a closed set, we may assume that it is the complement of an open set of the form $\cup_{i \in \mathbb{N}}\left(u_{i}, v_{i}\right)$ where each $\left(u_{i}, v_{i}\right)$ is an open interval with rational endpoints.

Given any rational open interval $I$ containing an element of $C$ and any $n \in \mathbb{N}$, we can canonically arithmetically define two disjoint rational subintervals of $I$, each of which meets $C$ and has length less than $2^{-n}$. To see this, first note that if $C$ meets $I$, then since every point of $C$ is an accumulation point, there must be distinct elements $c_{0}$ and $c_{1}$ of $C$ which are also in $I$. Each of these is contained in arbitrarily small rational subintervals of $I$. Since we know such subintervals exist, we can make their selection canonical by fixing an enumeration of the rational subintervals of $I$ and selecting the first two sufficiently small disjoint intervals $\left(a_{0}, b_{0}\right)$ and $\left(a_{1}, b_{1}\right)$ such that no finite collection of the open intervals defining the complement of $C$ covers $\left[\left(3 a_{0}+b_{0}\right) / 4,\left(a_{0}+3 b_{0}\right) / 4\right]$ or $\left[\left(3 a_{1}+b_{1}\right) / 4,\left(a_{1}+3 b_{1}\right) / 4\right]$. This last condition is arithmetical and insures that 
$\left(a_{0}, b_{0}\right)$ and $\left(a_{1}, b_{1}\right)$ meet $C$. (We are allowed to apply the Heine-Borel theorem here, since we are working in $\mathrm{ACA}_{0}$.)

Using our subintervals, we can define a full binary tree $T$ with rational number node labels such that each infinite path corresponds to a distinct element of $C$. To do this, let $I_{\langle\rangle}=(-.1,1.1)$ and $r_{\langle\rangle}=1 / 2$. Given $I_{\sigma}$ that meets $C$, choose $I_{\sigma \frown 0}=\left(a_{0}, b_{0}\right)$ and $I_{\sigma^{\prime} 1}=\left(a_{1}, b_{1}\right)$ as in the preceding paragraph, using $\operatorname{lh}(\sigma)+1$ for $n$. Let $r_{\sigma>i}=\left(a_{i}+b_{i}\right) / 2$ for $i \in\{0,1\}$. Define the tree $T$ by $T=\left\{r_{\sigma} \mid \sigma \in 2^{<\mathbb{N}}\right\}$. Note that each infinite path through $T$ defines a rapidly converging Cauchy sequence. No real defined in this fashion is covered by any finite collection of the open intervals defining the complement of $C$. Thus, by another application of the Heine-Borel theorem, each path through $T$ defines a distinct element of $C$.

Finally, we can select a countable subset of $C$ by restricting our attention to those paths through $T$ whose corresponding $0-1$ sequences are eventually constantly 0 . For any $n$ and any such sequence, we can find a distinct sequence which agrees on the first $n$ entries. The corresponding reals will differ by no more than $2^{-n}$. Consequently, this countable subset of $C$ is dense in itself.

Our formalization of derived sequences (call characteristic systems in [3]) appears below. In practice, the linear ordering $A$ is actually a well ordering, and the pre-image of an element $\alpha \in A$ is the set of isolated points ejected at stage $\alpha$ in the transfinite construction of the usual derived sequence for the set.

Definition $\left(\mathrm{RCA}_{0}\right)$ Let $X$ be a countable subset of $\mathbb{R}$. A derived sequence for $X$ consists of a pair $\langle A, h\rangle$, where $A$ is a countable linear ordering and $h: X \rightarrow A$ is a surjection such that for all $x \in X$ and $a \in A, h(x)=a$ implies

(1) $\exists \epsilon>0 \forall y \in X(0<|x-y|<\epsilon \rightarrow h(y)<a)$, and

(2) $\forall b<a \forall \epsilon>0 \exists y \in X(0<|x-y|<\epsilon \wedge h(y) \geq b)$.

If $X$ is a definable subset of $[0,1]$, we can define $\bar{X}$ to be the set of all open intervals with rational endpoints that contain no points of $X$. Depending on the defining formula of $X$, proving the existence of (the countable code) for $\bar{X}$ may require very strong comprehension axioms. Subject to the restriction that $\bar{X}$ exists, we can prove that exactly one of $\mathrm{W}(X)$ and $\mathrm{S}(X)$ holds. The reversal in the following theorem shows that the use of $A T R_{0}$ is unavoidable. 
Theorem $4\left(\mathrm{ACA}_{0}\right)$ The following are equivalent:

1. $\mathrm{ATR}_{0}$.

2. If $X$ is a subset of $[0,1]$ and $\bar{X}$ exists, then exactly one of $\mathrm{W}(X)$ and $\mathrm{S}(X)$ holds.

3. For every closed subset of $[0,1]$, exactly one of $\mathrm{W}(X)$ and $\mathrm{S}(X)$ holds.

Proof To prove that (1) implies (2), assume $\mathrm{ATR}_{0}$ and suppose $X \subseteq[0,1]$ and $\bar{X}$ exists. Either $\bar{X}$ is countable or $\bar{X}$ is uncountable.

First, suppose $\bar{X}$ is uncountable. Clearly, $X$ is not contained in any countable closed set, so $\mathrm{W}(X)$ fails. By Theorem V.4.3 of [5], from $\mathrm{ATR}_{0}$ we may deduce the perfect set theorem. Consequently, $\bar{X}$ contains a perfect set. By Lemma 3, we have that $\mathrm{S}(X)$ holds.

Now suppose that $\bar{X}$ is countable. Let $\bar{X}=\left\{x_{0}, x_{1}, \ldots\right\}$. Suppose, by way of contradiction, that $\bar{X}$ contains a countable dense in itself set. We can successively choose points $y_{i}$ in the dense in itself set and rationals $r_{i}>0$ so that for every $i<j$, $\left|y_{i}-y_{j}\right|<2^{-i}$ and $\left|y_{j}-x_{i}\right|>r_{i}$. The sequence $\left\langle y_{i}\right\rangle_{i \in \mathbb{N}}$ converges to an element of $\bar{X}$, but that element cannot be equal to any $x_{i}$, yielding the desired contradiction. Thus $\mathrm{S}(X)$ fails in this case. It remains to show that $\mathrm{W}(X)$ holds. Since $\bar{X}$ is a countable closed subset of $\mathbb{R}$, by Friedman and Hirst's [3] Lemma 4.4, $\bar{X}$ has a derived sequence; call it $\langle A, h\rangle$. By Lemma 4.2 of [3] we can assume $A$ is a well ordering. Note that $h$ maps $\bar{X}$ onto $A$. Define $f: \bar{X} \rightarrow A \times \mathbb{N}$ by $f\left(x_{i}\right)=\left(h\left(x_{i}\right), i\right)$. Let $Y$ denote the range of $f$. We say that $\left(a_{1}, i\right)<\left(a_{2}, j\right)$ if $a_{1}<a_{2}$ or both $a_{1}=a_{2}$ and $i<j$. Since $f$ is a bijection between $\bar{X}$ and $Y$, it defines a well-ordering of $\bar{X}$. It remains to show that if $C \subseteq X$ is closed then $f(C)$ has a maximum element. Fix $C$ and let $I=\{(a, i) \in Y \mid \forall x \in C f(x) \leq(a, i)\}$. By way of contradiction, suppose that $I=\emptyset$. Define a sequence $\left\langle x_{j_{i}}\right\rangle_{i \in \mathbb{N}}$ such that $f\left(x_{j_{i}}\right)$ is unbounded in $Y$. By the Bolzano Weierstraß theorem (which is provable in ACA ), we know that $\left\langle x_{j_{i}}\right\rangle_{i \in \mathbb{N}}$ contains a subsequence that converges to an element $x_{i}$ of $\bar{X}$. By the definition of derived sequence, $f\left(x_{i}\right)$ must exceed every element of $Y$, yielding a contradiction. Thus $I$ is not empty. Since $I$ is a nonempty subset of the well-ordering $Y$, $I$ has a minimum element; call it $\mu$. If $\mu \notin f(C)$ then we can define another sequence $\left\langle x_{k_{i}}\right\rangle_{i \in \mathbb{N}}$ such that $f\left(x_{k_{i}}\right)$ is unbounded in the initial segment of $Y$ below $\mu$. As above, this leads to a contradiction. Thus, $\mu$ is the maximum element of $f(C)$. Since $\mu$ is arithmetically definable from the code for $C, \mathrm{ACA}_{0}$ proves the existence of the function $g$ required for $\mathrm{W}(X)$.

Since (3) is a special case of (2), we can complete the proof by showing that (3) implies (1). Assume $A_{C A}$ and (3). We will use Theorem V.5.5 of Simpson's book [5], which 
says that $A T R_{0}$ can be derived from the statement that every $0-1$ tree with uncountably many infinite paths must contain an infinite perfect subtree. Let $T$ be a $0-1$ tree with uncountably many infinite paths. We will define $C$, a closed subset of the Cantor middle third set corresponding to the infinite paths of $T$. Emulating the proof of Theorem IV.1.2 of [5], for each $\sigma \in 2^{\mathbb{N}}$, define:

$$
a_{\sigma}=\sum_{i<\operatorname{lh}(\sigma)} \frac{2 \sigma(i)}{3^{i+1}} \quad b_{\sigma}=a_{\sigma}+\frac{1}{3^{\ln (\sigma)}} \quad a_{\sigma}^{\prime}=a_{\sigma}-\frac{1}{3^{\ln (\sigma)+1}} \quad b_{\sigma}^{\prime}=b_{\sigma}+\frac{1}{3^{\ln (\sigma)+1}}
$$

Let $C$ be the complement of the middle third intervals $\cup_{\sigma \in 2^{\mathbb{N}}}\left(b_{\sigma^{-0}}, a_{\sigma^{-1}}\right)$ together with the "leaf" intervals $\left(a_{\sigma^{\prime} \epsilon}^{\prime}, b_{\sigma^{-} \epsilon}^{\prime}\right)$ where $\epsilon \in\{0,1\}$ and $\sigma \in T$ but no extension of $\sigma$ lies in $T$. Clearly $C$ is closed. Since $T$ has uncountably many infinite paths, $C$ is uncountable. Thus $\mathrm{W}(C)$ must fail. By (3), $\mathrm{S}(C)$ must hold. Let $D=\left\{d_{0}, d_{1}, \ldots\right\}$ be a countable dense in itself subset of $C$. The set $D$ can be used to define a perfect subtree of $T$ as follows. Choose $\sigma_{\langle\rangle} \in T$ such that $d_{0} \in\left[a_{\left.\sigma_{\langle\rangle}\right\rangle}, b_{\left.\sigma_{\langle\rangle}\right\rangle}\right]$. Given $\sigma_{\tau}$ with $d_{i} \in \sigma_{\tau}$, let $\sigma_{\tau^{\wedge} 0}$ and $\sigma_{\tau^{\wedge} 1}$ be the first incompatible extensions of $\sigma_{\tau}$ such that there are integers $i_{0}$ and $i_{1}$ such that $d_{i_{0}} \in\left[a_{\sigma_{\tau^{\sim} 0}}, b_{\sigma_{\tau \sim 0}}\right]$ and $d_{i_{1}} \in\left[a_{\sigma_{\tau \sim 1}}, b_{\sigma_{\tau \sim 1}}\right]$. Such extensions must exist because $D$ is a dense in itself subset of $C$. The set of nodes $\left\{\sigma_{\tau} \mid \tau \in 2^{<\mathbb{N}}\right\}$ form a perfect subtree of $T$.

We now present the model theoretic result mentioned in the introduction. This theorem can be viewed as a partial converse for Theorem 1 and Theorem 2 .

Theorem 5 Suppose $X$ is a computable subset of [0,1]. If every $\omega$-model of $\mathrm{RCA}_{0}$ models $\mathrm{HB}(X)$, then $\langle\omega, \mathcal{P}(\omega)\rangle \models \mathrm{W}(X)$. If every $\omega$-model of $\mathrm{RCA}_{0}$ models $\mathrm{HB}(X) \rightarrow \mathrm{WKL}_{0}$, then $\langle\omega, \mathcal{P}(\omega)\rangle \models \mathrm{S}(X)$.

Proof Suppose $X$ is computable. To prove the first implication, suppose that every $\omega$-model of $\mathrm{RCA}_{0}$ models $\mathrm{HB}(X)$ and, by way of contradiction, that $\langle\omega, \mathcal{P}(\omega)\rangle \forall \mathrm{W}(X)$. Since $\langle\omega, \mathcal{P}(\omega)\rangle$ is a model of $\operatorname{ATR}_{0}$, by Theorem 4 we must have $\langle\omega, \mathcal{P}(\omega)\rangle=\mathrm{S}(X)$. Suppose $Y$ is a (code for a) countable dense in itself set that is contained in every closed superset of $X$. Let $U_{Y}=\left\{Z \subseteq \omega \mid Z \leq_{T} Y\right\}$. Then $\left\langle\omega, U_{Y}\right\rangle$ is an $\omega$-model of $\mathrm{RCA}_{0}$ and consequently, a model of $\mathrm{HB}(X)$. Since $Y$ is in $U_{Y},\left\langle\omega, U_{Y}\right\rangle \models \mathrm{S}(X)$. By Theorem 2, $\left\langle\omega, U_{Y}\right\rangle \models \mathrm{S}(X) \rightarrow \mathrm{WKL}_{0}$, so $\left\langle\omega, U_{Y}\right\rangle \models \mathrm{WKL}_{0}$. However, there is a tree computable from $Y$ with no $Y$-computable paths, so $\left\langle\omega, U_{Y}\right\rangle$ is not a model of $\mathrm{WKL}_{0}$.

We will prove the contrapositive of the second implication. Suppose that $\langle\omega, \mathcal{P}(\omega)\rangle \not \models$ $\mathrm{S}(X)$. Since $\langle\omega, \mathcal{P}(\omega)\rangle \models A \mathrm{ATR}_{0}$, by Theorem 4 we have $\langle\omega, \mathcal{P}(\omega)\rangle \models \mathrm{W}(X)$. By $\mathrm{W}(X)$, the closure of $X$ is a countable closed set. Consequently, $\langle\omega, \mathcal{P}(\omega)\rangle \models \mathrm{W}(\bar{X})$. Let $Y$ be (a code for) the associated well ordering and function. Define $U_{Y}$ as in the 
preceding paragraph. Then $\left\langle\omega, U_{Y}\right\rangle \models \mathrm{W}(\bar{X})$, and by Theorem $1,\left\langle\omega, U_{Y}\right\rangle \models \mathrm{HB}(\bar{X})$. Since every closed subset of $X$ is a closed subset of $\bar{X},\left\langle\omega, U_{Y}\right\rangle \models \mathrm{HB}(X)$. As above, $\left\langle\omega, U_{Y}\right\rangle \not \forall \mathrm{WKL}_{0}$, so $\left\langle\omega, U_{Y}\right\rangle$ is an $\omega$-model of $\mathrm{RCA}_{0}$ that does not model $\mathrm{HB}(X) \rightarrow \mathrm{WKL}_{0}$, as desired.

Over $\mathrm{RCA}_{0}$, the statement $\mathrm{HB}([0,1])$, which addresses open covers of arbitrary closed subsets of $[0,1]$, is easily deduced from the restriction of the statement to covers of the entire interval $[0,1]$. Thus, $\mathrm{WKL}_{0}$ can be deduced from the Heine-Borel Theorem restricted to a single closed set. Although $\mathrm{WKL}_{0}$ can be deduced from $\mathrm{HB}([0,1] \cap \mathbb{Q})$, there is no countable closed set for which the Heine-Borel Theorem implies $\mathrm{WKL}_{0}$. This can be proved as follows by utilizing the constructions from the proofs of Theorems 4 and 5.

Theorem 6 Suppose $X$ is a countable closed subset of $[0,1]$. Then there is a model $\mathcal{M}$ containing $X$ such that $\mathcal{M}=\mathrm{RCA}_{0}, \mathcal{M}=\mathrm{HB}(X)$, and $\mathcal{M} \models \neg \mathrm{WKL}_{0}$.

Proof Let $X$ be a countable closed subset of $[0,1]$. Since $X$ is (encoded) in $\langle\omega, \mathcal{P}(\omega)\rangle$, $\langle\omega, \mathcal{P}(\omega)\rangle \models \mathrm{ATR}_{0}$, and $\bar{X}=X$ is countable, we can emulate the third paragraph of the proof of Theorem 4 to find $F, f, g$, and $Y$ witnessing $\mathrm{W}(X)$. Choose a set $B$ such that $X, F, f, g$, and $Y$ are all computable from $B$, and let $U_{B}=\left\{Z \subseteq \omega \mid Z \leq_{T} B\right\}$. As in the proof of Theorem $5,\left\langle\omega, U_{B}\right\rangle$ is a model of $\mathrm{RCA}_{0}, \mathrm{HB}(X)$, and $\neg \mathrm{WKL}_{0}$.

The formulations of $\mathrm{W}(X)$ and $\mathrm{S}(X)$ are certainly not unique. It would be interesting to find other formulations that would satisfy Theorem 1, Theorem 2, and Theorem 5, but for which Theorem 4 could be carried out in a weaker system. On the other hand, the current formulations seem to have some applicability. For example, Hirst's [4] result showing that the Heine-Borel Theorem for $\mathbb{Q} \cap[0,1]$ implies $\mathrm{WKL}_{0}$ is an immediate consequence of Theorem 2. Also, Friedman and Hirst [3] define a canonical embedding of a well ordered set into $[0,1]$. Working in $\mathrm{RCA}_{0}$, if we can show that $X \subseteq[0,1]$ is a countable closed set and is the range of this sort of canonical embedding of a well ordering, then Theorem 1 shows that the Heine-Borel Theorem holds for $X$.

\section{Acknowledgments}

Portions of the Jeffry Hirst's work were supported by a grant (ID\# 20800) from the John Templeton Foundation. The opinions expressed in this publication are those of the authors and do not necessarily reflect the views of the John Templeton Foundation. Portions of the Jessica Miller's work were supported through the Graduate Research Associate Mentoring Program funded by the Cratis D. Williams Graduate School at Appalachian State University. 


\section{References}

[1] D K Brown, Notions of closed subsets of a complete separable metric space in weak subsystems of second-order arithmetic, in: Logic and Computation (Proceeding of Workshop at Carnegie-Mellon University, 1987), Contemp. Math. 106, Amer. Math. Soc., Providence, RI, 1990, 39-50.

[2] H M Friedman, Systems of second order arithmetic with restricted induction, I, II (abstracts), J. Symbolic Logic 41 (1976), 557-559.

[3] H M Friedman, J L Hirst, Reverse mathematics and homeomorphic embeddings, Ann. Pure Appl. Logic 54 (1991), 229-253; doi:10.1016/0168-0072(91)90048-Q.

[4] J L Hirst, A note on compactness of countable sets, in: Reverse mathematics 2001, Lect. Notes Log. 21, Assoc. Symbol. Logic, La Jolla, CA (2005), 219-221.

[5] SG Simpson, Subsystems of Second Order Arithmetic, second edition, Perspectives in Logic, Cambridge University Press, Cambridge, 2009; doi: 10.1017/CBO9780511581007.

Department of Mathematical Sciences, Appalachian State University, Boong, NC 28608

Department of Mathematics, Catawba Valley Community College, 2550 US Highway 70 SE, Hickory, NC 28602-8302

jlh@math.appstate.edu, jmiller@cvcc.edu

http: //www.math. appstate.edu/ jlh

Received: 9 June 2011 Revised: 18 January 2012 